\title{
Modulation of cochlear tuning by low-frequency sound
}

\author{
J.F.L. Klis ${ }^{1, *}$, V.F. Prijs ${ }^{2}$, J.B. Latour ${ }^{1}$ and G.F. Smoorenburg ${ }^{1}$ \\ 'Department of Otorhinolaryngology, University Hospital, Utrecht, The Netherlands and ' Department of Otorhinolaryngology. \\ University Hospital, Leiden, The Netherlands
}

(Received 19 January 1988; accepted 8 July 1988)

\begin{abstract}
An intense, low-frequency tone (about $30 \mathrm{~Hz}$ ) modulates the sensitivity of the inner ear to high-frequency stimulation. This modulation is correlated with the displacement of the basilar membrane. The findings suggest that the modulation may also affect cochlear tuning. We have investigated modulation of cochlear tuning by low-frequency sound in the guinea pig. Applying indirect methods of measurement (narrow-band analysis of compound action potentials and compound-action-potential tuning curves), the results suggest a shift of the excitation pattern along the basilar membrane towards higher-frequency areas. The shift occurred for both scala tympani and scala vestibuli displacement of the cochlear partition. Tuning curves, obtained from single units in the cochlear nerve, show sensitivity loss and a tip shift towards lower frequencies. This was also found for both scala tympani displacement and scala vestibuli displacement. The shift of the tip of the tuning curve towards lower frequencies corresponds to the inferred high-frequency shift of the excitation pattern. The relationship of these phenomena with the pathophysiology of Ménière's disease and with possible active mechanisms in cochlear transduction is discussed.
\end{abstract}

Low-frequency modulation: Narrow-band analysis; Compound-action-potential tuning curves: Single-unit tuning curves

\section{Introduction}

In a mechanical model of the inner ear, Tonndorf (1957) was able to show that an increase of endolymphatic pressure leads to loss of sensitivity and a shift of the maximum in the travelling-wave envelope towards the base. A broadening of the envelope was also observed. Tonndorf suggested a link between the shift in the travelling-wave envelope and binaural diplacusis, which is often found in Ménière's disease. Crucial to this hypothesis is, that endolymphatic overpressure, leading to permanent displacement of the basilar membrane, is associated with endolymphatic hydrops, found in Ménière's disease (Hallpike and Cairns, 1938; Schuknecht, 1974).

Correspondence to: G.F. Smoorenburg, Department of Otorhinolaryngology, University Hospital, Catharijnesingel 101, 3511 GV Utrecht, The Netherlands.

* Present address: Department of Veterinary Pharmacology, Pharmacy and Toxicology, University of Utrecht, P.O. Box $80.176,3508$ TD Utrecht, The Netherlands.
The experimental approach to the problem of Ménière's disease has mainly been directed at introducing an artificial endolymphatic hydrops in animals (Aran et al., 1984; Morgenstern, 1985; Morizono et al., 1985; Van Deelen et al., 1987; Klis and Smoorenburg, 1988). Some of the effects, observed by Tonndorf in his mechanical model after increasing the endolymphatic pressure were also observed in this experimental hydrops model. Electrophysiologically, Harrison et al. (1984) and Harrison and Prijs (1984) found loss of sensitivity and broadening of cochlear tuning in hydropic ears of guinea pigs. It is interesting to note, that the same effects are found in Ménière patients, both psychophysically (Formby, 1986) and electrophysiologically (Harrison et al., 1985). It is, however, not clear that the effects found in hydropic guinea pigs are due to the hydrops itself (increase of the endolymphatic space with possible endolymphatic overpressure). Albers et al. (1987). for example, found considerable outer hair cell loss 4 and 8 months after introducing the hydrops. This may have affected the electrophysiological 
results collected by both Harrison et al. (1984) and Harrison and Prijs (1984) at about one year after hydrops was introduced. The hydrops model is also not well suited to investigate a possible shift of the travelling-wave envelope, as found by Tonndorf (1957), because an immediate comparison of results before and during hydrops is impossible. For example, a tuning curve can not be measured in the same nerve fiber before and during hydrops. Therefore, we thought of other methods to study the effect of basilar-membrane displacement, assumed to occur with endolymphatic overpressure, on cochlear tuning.

A suitable candidate is the low-frequency biasing technique. With this method, the system is tested while probe sounds are presented during specific phases of a very-low-frequency tone (about $30 \mathrm{~Hz}$ ), i.e. during specific quasi-static displacements of the basilar membrane. The term quasistatic is used, because a sinusoidal bias has both displacement and velocity components, that constantly vary in time. Applying this technique, several interesting parallels with Ménière's disease were found, like enhancement of the summating potential with scala tympani displacement (Klis and Smoorenburg, 1985).

Patuzzi et al. $(1984 a, b, c)$ found a decrease in unit firing rate during both bias-evoked scala tympani displacement and scala vestibuli displacement. This reduction was most pronounced for scala tympani displacement, while stimulating at the characteristic frequency. They were able to track down this effect to a reduction in mechanical sensitivity of the basilar membrane. They suggested that the reduction in the sensitivity was due to disturbance of a nonlinear process in basilar membrane motion. Such a process might be described by a negative damping, as has been suggested by Kim et al. (1980) and Neely and Kim (1983). Although this model is still speculative (Schmiedt (1982), for example, did not find a decrease of sensitivity with scala tympani displacement), we expect that the same type of disturbances may play a role in Ménière's disease and hydrops if basilar membrane displacement is associated with these disorders.

In conclusion, the low-frequency biasing technique seems to provide us with a reasonably satisfying method to study the effects of basilar mem- brane displacement in relation to hearing disorders, found in Ménière's disease. At the same time it provides us with information on basic mechanisms, involved in basilar membrane motion. In this paper we shall investigate possible shifts in cochlear tuning with basilar membrane displacements, using the biasing technique in guinea pigs. The results will be compared with the binaural diplacusis phenomenon in Ménière patients.

We shall use three methods to evaluate cochlear tuning. The first one is narrow-band analysis of compound-action-potentials (AP). With this technique contributions to the AP from restricted frequency regions along the cochlear partition are determined, by selectively masking the contributions from the other frequency regions (Teas et al., 1962; Prijs and Eggermont, 1981). In combination with the biasing technique, we might be able to detect shifts in the region contributing most to the $\mathrm{AP}$. The second technique is the AP-tuning-curve technique. It is based on masking the response to a low-level probe tone stimulating a restricted set of adjacent nerve fibers (Dallos and Cheatham, 1976; Van Heusden and Smoorenburg, 1981). Both techniques, narrow-band analysis and AP-tuning curves, are based on masking. This implies, that possible effects of low-frequency biasing may not show up, because the bias may affect both the masker and the probe tone or, when the masker precedes the probe tone, the results will be difficult to interpret, because the masker is affected at another phase of the bias as the probe. We tried to minimize this problem by prescnting the masker during at least a full period of the low-frequency bias. After we found an effect of low-frequency biasing on tuning with these macro-electrode techniques, the final check was made with the third technique, the single-unit tuning curve technique (e.g. Evans, 1979).

\section{Materials and methods}

\section{Narrow-band analysis}

In two young, healthy, albino guinea pigs the narrow-band analysis technique was applied to round-window responses to $8 \mathrm{kHz}$ tone bursts, positioned at different phases of a low-frequency bias. Details of animal anaesthesia and prepara- 
tion have been described before (Klis and Smoorenburg, 1985) and will not be discussed here.

Stimulus generation and data acquisition were fully controlled by a PDP 11-23 minicomputer system. The tone burst was calculated point by point and stored in a revolving memory containing 1024 points with 12 bit resolution. Its rise- and fall-envelopes were cosine shaped ( 4 periods each) and it had a plateau of 32 periods. The lowfrequency bias stimulus was generated by a Rockland programmable sine generator and was held constant at $29 \mathrm{~Hz}$. Distortion, measured in $1 / 3$ octave filters, phase locked to the bias, was at least $70 \mathrm{~dB}$ down with respect to the fundamental. A timer, locked to the bias, served to release the probe tone at a specified phase of the lowfrequency bias. The masking noise, required for the narrow-band analysis, was produced by a Wandel und Goltermann noise generator and had a flat spectrum between $16 \mathrm{~Hz}$ and $20 \mathrm{kHz}$, which may be considered 'white' for our purposes. For adjustable high-pass filtering of the noise, two programmable Rockland filters were used in series, together producing a high-pass slope of $96 \mathrm{~dB} /$ oct. The noise was fed to a Beyer DT48 dynamic telephone, while the combination of probe and bias was fed into a second DT48 telephone.

The two telephones were connected to a hollow ear bar, which was inserted into the external auditory meatus of the animal. Care was taken to avoid air leakage, which dramatically deteriorates the low-frequency output of the sound delivery system. The noise had to be shaped by a custombuilt system to obtain a reasonably flat spectrum (variation within $10 \mathrm{~dB}$ between 4 and $10 \mathrm{kHz}$ ) at the outlet of the ear bar. Calibration was performed, using the substitution method with a half-inch condenser microphone (Brïel and Kjaer 4134 with 2619 follower) at the approximate site of the tympanic membrane.

The experiment was performed using a forward-masking paradigm, to avoid direct interaction of the masker and the probe (e.g. two-tone suppression). The temporal structure of the stimulus for one phase in the low-frequency bias is depicted in Fig. 1.

The round-window responses were amplified 10000 times, bandpass filtered between $1 \mathrm{~Hz}$ and $10 \mathrm{kHz}$ and led to a 12 bit AD-converter (Data Translation DT2784). Averaging was usually based on between 100 and 500 samples. The averaged responses were stored on hard disc for off-line analysis.

The protocol was hased on the work of Teas et al. (1962) and Prijs and Eggermont (1981). First, the threshold to the probe tone is determined. Next, the intensity level of the unfiltered noise is fixed at a level, which just masks the probe-tone evoked response, with tone-burst level, in our case, somewhere between 10 and $40 \mathrm{~dB}$ above threshold. In successive recordings the cut-off frequency of the high-pass filter is increased stepwise (cut-off frequencies at $4,5,6,8,11,15,20 \mathrm{kHz}$ ). Subtraction of two successive registrations is assumed to produce the response from a specific region of the basilar membrane: a Narrow-Band AP (NAP). A plot of the amplitude of the NAP versus the geometric-center frequency of the 'pass-band', bounded by two successive cut-off frequencies

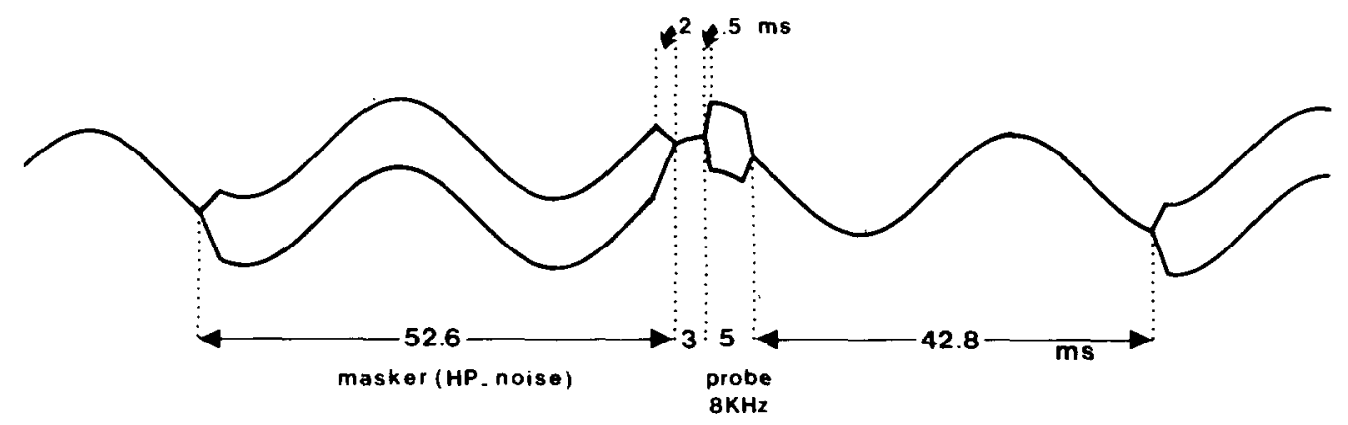

Fig. 1. Timing sequence of the stimulus, used in narrow-band analysis. A high-pass filtered noise masker precedes an $8 \mathrm{kHz}$ probe tone, placed at a specific phase, in this case $90^{\circ}$, of a continuous low-frequency bias $(29 \mathrm{~Hz}$ ). 
yields an excitation pattern (e.g. Fig. 4). In our case, the NAP amplitude is expressed as a percentage of the mean of two control responses, one determined before and one after NAP gathering. These control responses were measured at the same phase of the bias, but without masking noise. This procedure was repeated for each phase. The phase of movement of the basilar membrane was inferred from the low-frequency cochlear microphonic at the round window, using data from Sellick et al. (1982).

As already mentioned, an essential presumption is, that the bias exerts its effect mainly on the short tone burst and less on the masker. By choosing a relatively long masker duration, we hoped that the effect of the bias on the masker would, to some extent, average out.

\section{Compound action potential (AP) tuning curves}

Ten young, healthy, albino guinea pigs were used in this part of the experiment. Animal preparation, probe generation, bias generation and data acquisition were essentially the same as described above. A $4 \mathrm{kHz}$ probe (4 periods rise- and fall-time, 24 periods plateau) was used mostly. Occasionally, an $8 \mathrm{kHz}$ tone burst (4 periods riseand fall-time, 32 periods plateau) was used. The masking tone was produced by a Hewlett-Packard $3300 \mathrm{~A}$ sine wave generator, its frequency controlled by computer through its VCO-input. In order to obtain an acceptable distortion level, the sine wave was fed into four Rockland programmable filters, configured as a bandpass filter with skirts of $96 \mathrm{~dB} /$ oct. Upper harmonics were at least $70 \mathrm{~dB}$ down. A forward-masking paradigm was used. AP-tuning curves, obtained with a forward-masking paradigm are more narrow than those obtained with a simultaneous masking paradigm (van Heusden and Smoorenburg, 1981). The temporal structure of the stimulus at one phase of the low-frequency bias is depicted in Fig. 2. At high levels of the masker, a masker-evoked afterpotential appeared and hampered the measurements. Subtraction of the afterresponse, measured without a probe stimulus, from the compound response considerably facilitated the measurements.

The protocol was as follows. First, the threshold for the probe was measured. Probe level was then set at a moderate level above threshold, in order to excite a small, restricted region along the basilar membrane. Next, the level of the masker, required to reduce the $\mathrm{N}_{1}$ action-potential amplitude to $50 \%$ of its unmasked value was determined by linear interpolation of $\mathrm{N}_{1}$-amplitude versus masker level at each masker frequency. A plot of the $50 \%$ level versus masker frequency yielded the AP-tuning curve (e.g. Fig. 5). This procedure was performed without bias and with bias, while probe onset was locked at inferred maximum displacement of the basilar membrane towards scala tympani and towards scala vestibuli. When the bias was present, $50 \%$ reduction was defined with respect to the amplitude of the probe response, measured without masking, but with bias.

The same presumption, which had to be made for the narrow-band analysis, must be made here. The bias exerts its effect mainly at the probe

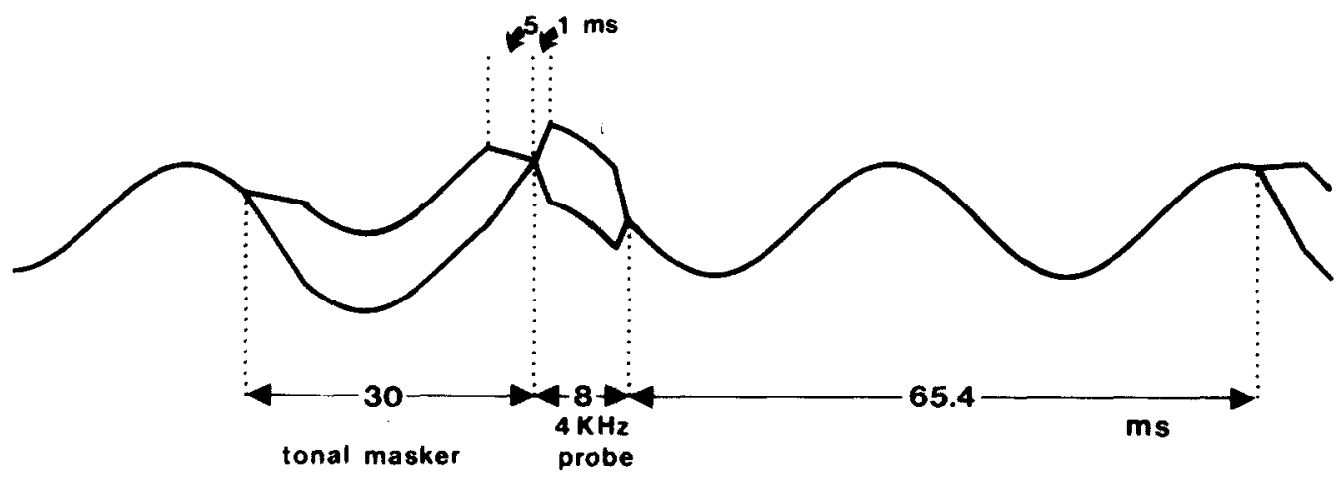

Fig. 2. Timing sequence of the stimulus, used in the AP-tuning experiment. A pure tone masker precedes an 8 or $4 \mathrm{kHz}$ probe tone, placed at a specific phase, in this case $90^{\circ}$, of a continuous low-frequency bias $(29 \mathrm{~Hz})$. 
response, while it affects the relatively long masker to a smaller degree.

\section{Tuning determined from single-unit responses}

Fifteen young, healthy, albino guinea pigs were used for this part of the investigation. Animal preparation, test tone stimulation and data acquisition were basically the same as described in Harrison and Prijs (1984) and will not be discussed here. The bias stimulus was mixed electrically with the test tone stimulus. Bias distortion products were at least $50 \mathrm{~dB}$ down with respect to the fundamental in the frequency range where the measured fibers have their best frequency.

The method used to determine the frequency tuning curve was based on the threshold-tracking method (Evans, 1979). In order to save time, the method of applying the threshold-tracking paradigm was different in control situations and when the bias was present. In the control experiment the number of spikes fired in a time window of $50 \mathrm{~ms}$ during test-frequency stimulation is compared with the number of spikes during a time window of the same length, but without stimulation. The test level was decreased or increased in steps of $1 \mathrm{~dB}$, while test frequency was decreased in steps of $1 / 50$ th of an octave. The threshold criterion was set at $50 \%$ increase with respect to the response, measured when no test tone was present. When the bias stimulus $(31.25 \mathrm{~Hz}$, period: $32 \mathrm{~ms}$ ) was present, the time window was divided into 6 windows of $8 \mathrm{~ms}$ duration each, the windows positioned in successive periods of the bias and centered at maximum displacement of the cochlear partition towards either scala tympani or scala vestibuli. Again. the phase of displacement of the basilar membrane was inferred from the lowfrequency cochlear microphonic, measured at the round window. The number of spikes fired in the 6 sub-windows during test-frequency stimulation was compared with the number of spikes in the 6 sub-windows when test frequency was off (Fig. 3). Unavoidably, this method consumes at least 4 times as much time as the control measurements in an already time critical experiment. We chose 8 $m s$ for the duration of the sub-windows as a compromise between phase specificity and time efficiency.

\section{DETECTION WINDOWS, 8 MS EACH}

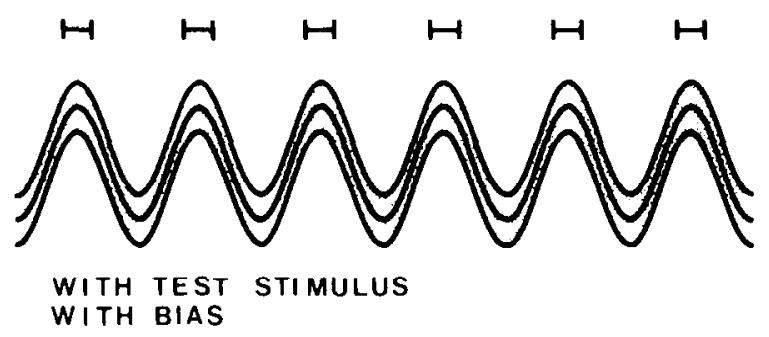

6 DETECTION WINDOWS, 8 MS EACH

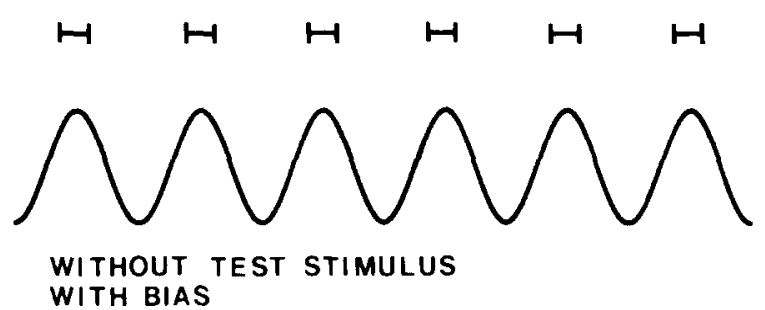

Fig. 3. Stimulus configuration for the threshold-tracking paradigm, used in the single-unit experiment. Six successive detection windows, each $8 \mathrm{~ms}$ in duration, are centered around a specific phase of a continuous low-frequency bias $(31.25 \mathrm{~Hz})$. The spike count in the 6 windows, when a test-tone stimulus is present, is compared with the count in the 6 windows, when only the bias stimulus is present.

It is important to note, that different results are to be expected from the different methods. A frequency shift, measured with the indirect masking methods, will be in the opposite direction from a frequency shift measured with the direct method, the single-unit method. The indirect methods give information about the population of fibers contributing to the compound response. An upward shift in this population implies, that an individual fiber will respond best when the stimulus frequency is shifted into a downward direction.

\section{Results}

\section{Narrow-band analysis}

A clear example of the effect of the bias on the narrow-band APs is depicted in Fig. 4. Biasing suppresses the AP in a phase-dependent manner. The relative magnitude of this effect increases as 


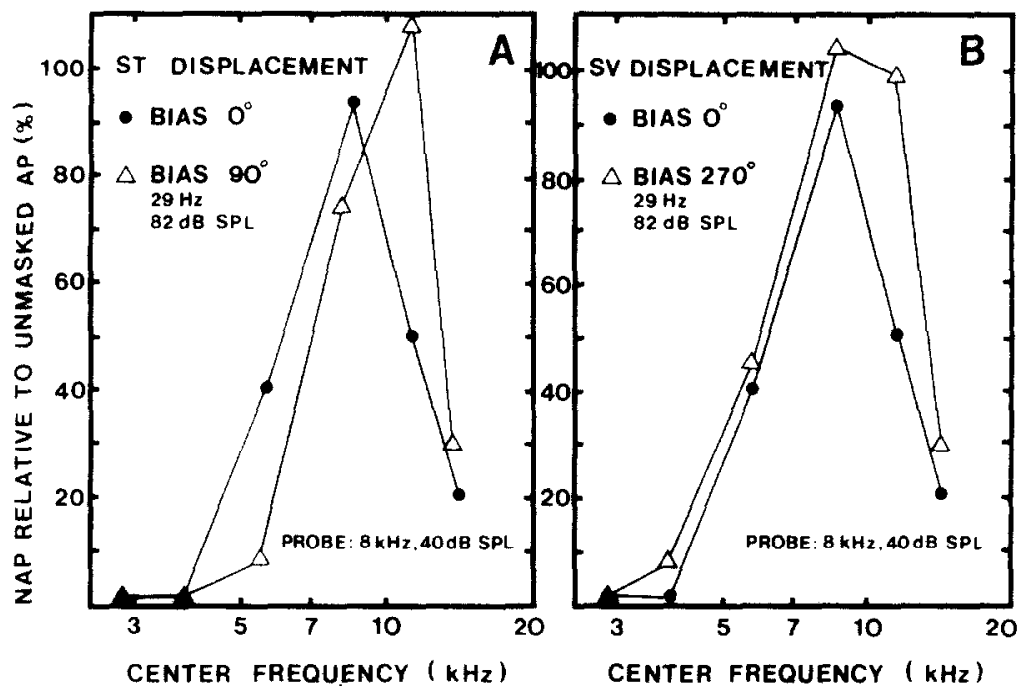

Fig. 4. Typical example of a result from narrow-band analysis. The narrow-band AP (NAP) is expressed in percents, relative to the AP, measured without a noise masker, but with bias. The NAP is plotted versus the center frequency of the relevant pass band. This plot reflects the excitation pattern on the basilar membrane. The excitation pattern without basilar membrane displacement (closed circles) is compared with the excitation pattern during scala tympani displacement (A) and scala vestibuli displacement (B). Note the shift of the excitation pattern towards higher frequencies and broadening of the pattern.

the probe level decreases (Klis and Smoorenburg, 1985). Thus, on the one hand, a medsurable probe response at all phases of the bias requires fairly high probe levels, on the other hand, low probe levels are required in order to excite restricted cochlear regions. A compromise between these two conflicting requirements was found by choosing a probe level of $40 \mathrm{~dB}$ SPL which is about 40 $\mathrm{dB}$ above AP threshold for $8 \mathrm{kHz}$. Still, for this level, the AP, especially when measured during scala tympani displacement, was very small(and its constituting NAPs even smaller). A considerable amount of uncertainty in determining the relative amplitude had to be accepted. This explains the fact, that relative amplitudes of more than $100 \%$ were sometimes encountered. In Fig. 4, the excitation pattern at 0 degrees (no displacement) is compared with that at 90 degrees (maximum scala tympani displacement, Fig. 4A) and 270 degrees (maximum scala vestibuli displacement, Fig. 4B). In spite of the complications described above, a shift of the excitation pattern towards higher frequencies is evident. For the displacement towards scala tympani, this shift was always found. For displacement towards scala vestibuli, it was not always as clear. Together with the pattern shift, a widening of the excitation pattern seems to be present.

\section{Compound action potential (AP) tuning curves}

In the AP-tuning experiment the same kind of problem was encountered as in the narrow-band analysis. We wanted to perform the experiment with the same probe level in all conditions. Hence, a probe level well above threshold was required, because of the suppressing effect of the bias at some phases, whereas the AP-tuning curve technique gives the best defined results just above threshold (Dallos and Cheatham. 1976: Van Heusden and Smoorenburg, 1981). These two conflicting requirements limit the range of probe levels in which the experiment can be performed. A level of $33 \mathrm{~dB}$ SPL turned out to be an acceptable compromise. The AP-tuning curves showed essentially the same effect as the narrow-band analysis: a tip shift towards higher frequencies. One of the clearest examples is shown in Fig. 5 for one animal. but comparable results were found in 9 other animals for both $4 \mathrm{kHz}$ and $8 \mathrm{kHz}$ probes. The control curve was measured without bias and is the same in Fig. SA and Fig. 5B. In general. the effect was most pronounced for scala tympani 

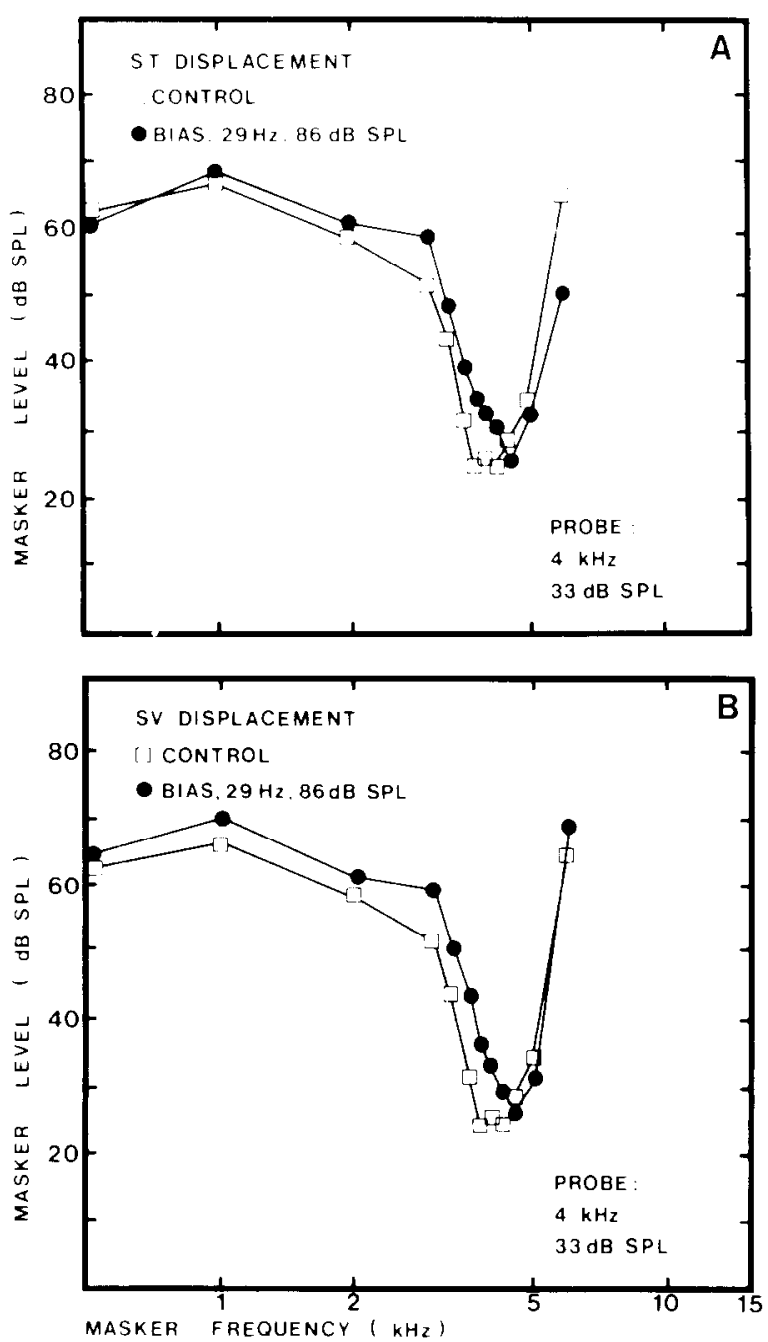

Fig. 5. AP-tuning curves, obtained with and without lowfrequency biasing. Masker level, needed to reduce the AP to $50 \%$ of its non-masked value is plotted against masker frequency. The control curve measured without bias (squares) is compared with the curve measured during maximum scala tympani displacement of the cochlear partition (A) and the one during maximum scala vestibuli displacement (B). Note shift of the curve towards higher frequencies.

displacement (Fig. 5A). A widening of the AP-tuning curve during basilar membrane displacement was not observed. On the contrary, the $Q_{10}$ values were sometimes even larger in the bias condition.

\section{Tuning determined from single unit responses}

We obtained reliable data from 20 fibers. As already pointed out in the Methods section. it takes rather a long time to obtain a tuning curve during low-frequency biasing. We frequently lost the fiber after completing measurement of the control tuning curve. but before completing the bias-experiment. Nevertheless, for some fibers, we were able to acquire as much as 6 tuning curves at several bias levels, during both scala tympani displacement and scala vestibuli displacement. In Fig. 6 all our data are shown together. Points represent the tip frequency (the characteristic frequency, CF) and level at the tip of the control tuning curve. squares and circles point to tip frequency and level of the tuning curve obtained during low-frequency biasing (squares towards scala tympani displacement, circles towards scala vestibuli displacement). Two major effects are apparent. Tip level increases and frequency at the tip decreases. Bias levels varied between 40 and 110 dB SPL. In general, lower bias levels were needed for fibers with a lower characteristic-frequency to obtain the same effect. There was no major difference between scala vestibuli displacement and scala tympani displacement, although scala tympani displacement appeared a little more effective.

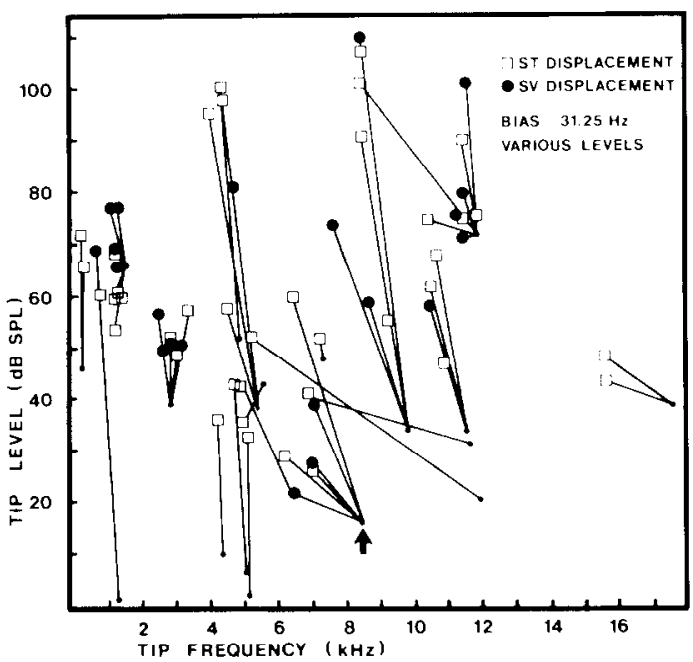

Fig. 6. Summary of the single unit results. Threshold level at the tip frequency is plotted against tip frequency. For each fiber, points represent the situation without bias, connected squares refer to the tip level and tip frequency at scala tympani displacement, connected circles refer to scala vestibuli displacement. Note sensitivity loss and tip shift towards lower frequencies. Bias frequency was $31.25 \mathrm{~Hz}$. Bias level varied. 

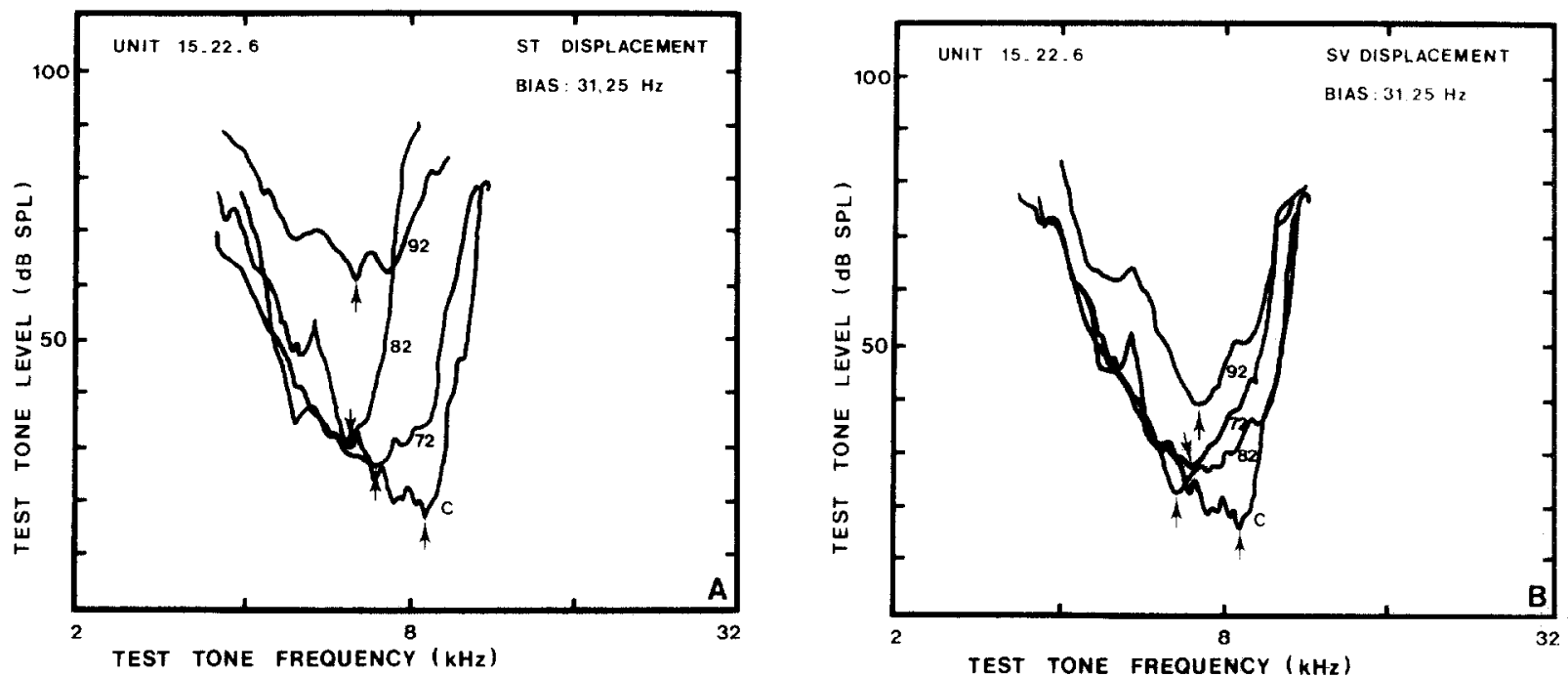

Fig. 7. Actual tuning curves from one fiber, obtained with and without bias. The control curve is compared with curves obtained during increasing scala tympani displacement (A) and scala vestibuli displacement (B). Bias frequency was $31.25 \mathrm{~Hz}$, bias level is indicated at each curve, $\mathrm{c}$ refers to the control curve. Note sensitivity loss, especially at the tip, and a shift of the tip towards lower frequencies. Also note nonmonotonic development of the frequency shift with increasing bias level.

As an example, Fig. 7 shows the actual tuning curves for one fiber indicated by the arrow in Fig. 6. For clarity, Fig. 7A shows the tuning curves obtained during scala tympani displacement and

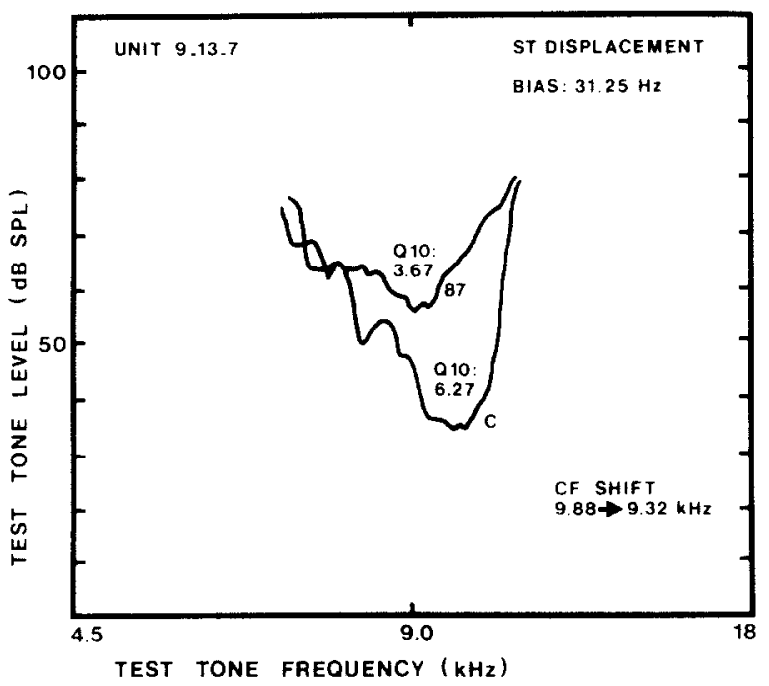

Fig. 8. Example of broadening of the tuning curve with lowfrequency biasing. The control curve (c) is compared with a curve obtained during scala tympani displacement of the cochlear partition. Bias frequency was $31.25 \mathrm{~Hz}$, bias level was $87 \mathrm{~dB}$ SPL. $O_{10}$ is indicated at each curve. It decreases dramatically in the bias condition. the control tuning curve, while Fig. 7B shows the scala vestibuli curves and the same control curve. The frequency shift seems to grow fairly monotonically with bias level, except at the highest level used. A reversal effect at very-high bias levels, where CF becomes higher again, was also seen in some other fibers. The level of the tip increases fairly monotonically with bias level, with the exception of the $82 \mathrm{~dB}$ SPL level in Fig. 7B. A monotonic growth of tip level with bias level was observed in nearly all other fibers, for which we were able to collect results at more than one bias level. The width of the tuning curve tended to increase in the bias condition, as can be seen in Fig. 8 for another nerve fiber. In this case, biasing towards scala tympani at an $87 \mathrm{~dB}$ SPL bias level leads to a reduction of $Q_{10}$ by nearly $50 \%$.

\section{Discussion}

In this study we have shown, that displacing the basilar membrane by applying a low-frequency bias sound interferes with the tuning mechanism of the cochlea. Data, collected according to three different methods, agree with one another on this matter. The indirect methods (narrow-band analysis and AP tuning) show us, that the excitation pattern on the basilar membrane shifts basal- 
wards, to higher nominal frequency areas. Hence, for the individual fiber, the frequency to which it responds best becomes lower, which is exactly what we have found in the single-unit experiments. It seems, that the presumption we had to make with regard to a smaller effect of the bias on the masker (whether it was high-pass noise or a pure tone) than on the probe is a valid one.

Sellick et al. (1982) observed suppression of evoked activity during both scala tympani and scala vestibuli displacement, when high bias levels were used. We found comparable effects. Sellick et al. (1982) found the largest effect at the fibers' characteristic frequency. This is compatible with our results, where the tip of the tuning curve is generally more affected than the tail (c.f. Fig. 8).

Suppression of evoked activity during lowfrequency biasing does not necessarily mean, that the tip of the tuning curve should shift upwards on the level axis. Single-unit tuning curves are plots of the probe tone level at which a certain rate criterion is met, versus probe frequency. Shifts in tuning may depend on the particular choice of criterion. In our case, the criterion is expressed as a fractional increase in the rate, found without probe-tone stimulation, but with biasing. Had we used an absolute increase in the number of spikes, regardless of the rate found without probe tone stimulation, the shift would probably have been greater.

The basalward shift of the excitation pattern during scala tympani displacement agrees with the model data from Tonndorf (1957). Scala vestibuli displacement, which was not investigated by Tonndorf, leads to similar results, though it seemed that here higher bias levels were needed to obtain the same effect. Patuzzi and Sellick (1984) presented tuning curves for DC-receptor potentials of one inner hair cell during low-frequency biasing. The curve for scala tympani displacement shows a definite tip shift toward a lower frequency. This is directly comparable with our results.

Ménière-patients report a higher perceived pitch in the affected ear (Tonndorf, 1957). In a simple tonotopic model for pitch perception this means that the stimulating tone evokes activity in fibers, which were normally tuned to higher frequencies or, in other words, a basalward shift of the excitation pattern. This is in line with our results.
A loss of frequency selectivity, comparable with our results $\left(Q_{10}\right)$ was found electrophysiologically in guinea pigs with an endolymphatic hydrops (Harrison and Prijs, 1984: Harrison et al., 1984). It could also be demonstrated psychophysically (Formby, 1986) and electrophysiologically (Harrison et al., 1985) in Ménière-patients. As mentioned already in the Introduction section. it should be kept in mind however, that deterioration of frequency selectivity can stem from a multitude of cochlear insults, not only basilar membrane displacement.

Some speculations can be made as to the mechanism through which (quasi-) static displacement of the basilar membrane exerts its effect on the transduction system. Kim et al. (1980) and Neely and Kim (1983) have published a mathematical model, which can serve to describe our results. In order to obtain sufficiently sharp and sensitive motion patterns of the basilar membrane, they had to postulate a negative damping process in the region of highest sensitivity for a certain stimulus frequency. This process contributes energy to the motion of the basilar membrane. Uncoupling of this process in the mathematical model leads to loss of sensitivity and a shift of the tip of the excitation pattern towards higher frequencies. These are essentially the same features as we have found in the present experiment. The hypothesis might be adopted, that displacement of the basilar membrane inactivates the undamping process. Circumstantial evidence supporting this hypothesis can be found in a study of Zwicker and Scherer (1987). They found, in humans, phase-dependent suppression of delayed otoacoustic emissions. evoked by $1300 \mathrm{~Hz}$ tone bursts which were riding on complex very-low-frequency sonic suppressors. The evoked otoacoustic emission is a product of an active process in the cochlea. The suppression was closely related to the second derivative of the sound pressure time function. According to Dallos (1970), in humans basilar membrane displacement follows the second derivative of sound pressure.

It is rather difficult to explain Tonndorf"s results (1957) on the basis of this model hecause no active processes were present in his mechanical model. We have to assume that. in his model, the damping increased, when the endolymphatic pressure was increased. This has the same effect as a 
reduction of the undamping component in the model of Kim et al. (1980).

An increase of the stiffness component, which intuitively seems a plausible effect of biasing, would lead to the opposite shift; the excitation pattern would move to lower-frequency regions, and individual fibers would respond best to higher stimulus frequencies. This might explain the results at high bias levels shown in Fig. 7, where the direction of the frequency shift reverses with respect to the direction found at lower bias levels.

\section{Acknowledgements}

The single-unit experiments were performed in the Department of Otorhinolaryngology (Head: Prof. Dr. P.H. Schmidt) from the University Hospital in Leiden, where Vera Prijs has her Laboratory. This research was supported by the Netherlands Organization for the Advancement of Pure Research (ZWO) and by the HeinsiusHoubolt Fund.

\section{References}

Albers, F.W.J.. Veldman, J.E. and Huizing, E.H. (1987) Early hair cell loss in experimental hydrops. Ann. Otol. Rhinol. Laryngol. 96, 282-285.

Aran, J.-M., Rarey, K.E. and Hawkins, J.E. (1984) Functional and morphological changes in experimental endolymphatic hydrops. Acta Otolaryngol. 97, 547-557.

Dallos, P. (1970) Low-frequency auditory characteristics: Species dependence. J. Acoust. Soc. Am. 48, 489-499.

Dallos, P. and Cheatham, M.A. (1976) Compound action potential (AP) tuning curves. J. Acoust. Soc. Am. 59 . $591-597$.

Evans, E.F. (1979) Single unit studies of mammalian cochlear nerve. In: H.A. Beagley (Ed.), Auditory Investigation. The Scientific And Technological Basis, Clarendon Press. Oxford, pp. 324-367.

Formby, C. (1986) Frequency and rate discrimination by Menière patients. Audiology 25, 10-18.

Halipike. C.S. and Cairns, H. (1938) Observations on the pathology of Ménière's syndrome. J. Laryngol. Otol. 53, $625-635$.

Harrison, R.V., Orsulakova, A., Erre, J.-P., Aran, J.-M., Morgenstern, C. and Tavartkiladze, G.A. (1984) Electrophysiological measures of cochlear function in guinea pigs with long-term endolymphatic hydrops. Hear. Res. 14 , $85-91$.

Harrison, K.V. and Prijs, V.F. (1984) Single cochlear fibre responses in guinea pigs with long-term endolymphatic hydrops. Hear. Res. 14, 79-84.
Harrison, R.V., Dauman, R., Tavartkiladze, (G.A and Aran, J.-M. (1985) Effects of glycerol on cochlear frequency selectivity in patients with Ménière's disease. I. Speech Hear. Disord. 50, 213-217.

Kim, D.O.. Neely, S.T., Molnar, C.E. and Matthews. J.W. (1980) An active cochlear model with negative damping in the partition comparison with Rhode's ante- and postmortem observations. In: G. van den Brink and F.A. Bilsen (Eds.). Psychophysical, Physiological and Behavioural Studies in Hearing, University Press. Delft. pp. 7.14.

Klis, J.F.L. and Smoorenburg, G.F. (1988) Cochlear potentials and their modulation by low-frequency sound in early endolymphatic hydrops. Hear. Res. 32, 175-184.

Klis, J.F.L. and Smoorenburg, G.F. (1985) Modulation at the guinea pig round window of summating potentials and compound action potentials by low-frequency sound. Hear. Res. 20, 15-23.

Morgenstern, C. (1985) Pathophysiologie, Klinik und konservative Therapie der Menièrische Erkrankung. Arch. Otorhinolaryngol. 241, S1, 1-66.

Morizono. T., Cohen, J. and Sikora, M.A. (1985) Measurement of action potential thresholds in experimental endolymphatic hydrops. Ann. Oto. Rhinol. Laryngol. 94. $191-194$

Neely, S.T. and Kim, D.O. (1983) An active cochlear model showing sharp tuning and high sensitivity. Hear. Res. 9. $123-130$.

Patuzzi, R., Sellick, P.M. and Johnstone, B.M. (1984a) The modulation of the sensitivity of the mammalian cochlea by low-frequency tones. I. Primary afferent activity. Hear. Res. 13, $1-8$.

Patuzzi, R. and Sellick, P.M. (1984b) The modulation of the sensitivity of the mammalian cochlea by low frequency tones. II. Inner hair cell receptor potentials. Hear. Res. 13, 9-18.

Patuzzi, R., Sellick, P.M. and Johnstone, B.M. (1984c) The modulation of the sensitivity of the mammalian cochlea by low-frequency tones. III. Basilar membrame motion. Heal. Res. 13, 19-27.

Prijs, V.F. and Eggermont, J.J. (1981) Narrow-band analysis of compound action potentials for several stimulus conditions in the guinea pig. Hear. Res. 4, 23-41.

Schmiedt, R.A. (1982) Effects of low-frequency biasing on auditory-nerve activity. J. Acoust. Soc. Am. 72, 142-150.

Schuknecht, H.F. (1974) Pathology of the ear. Harvard University Press, Cambridge, Massachusetts.

Sellick, P.M., Patuzzi, R. and Johnstone, B.M. (1982) Modulation of responses of spiral ganglion cells in the guinea pig cochlea by low-frequency sound. Hear. Res. 7, 199-221.

Teas, D.C., Eldredge, D.H. and Davis, H. (1962) Cochlear responses to acoustic transients: An interpretation of whole nerve action potentials. J. Acoust. Soc. Am. 34. 1438-1489.

Tonndorf. J. (1957) The mechanism of hearing loss in early cases of endolymphatic hydrops. Ann. Otol. Rhinol. Laryngol. 66, 766-784

Van Deelen, G.W., Ruding, P.R.W.J., Veldman, J.E., Huizing, E.H. and Smoorenburg. G.F. (1987) Electrocochleographic 
study of experimentally induced endolymphatic hydrops. Arch. Otorhinolaryngol. 244, 167-173.

van Heusden. E. and Smoorenburg. G.F. (1981) Eight-nerve action-potential tuning curves in cats before and after inducement of an acute noise trauma. Hear. Res. 5. 2548 .
Zwicker. E. and Scherer, A. (1987) Correlation between time functions of sound pressure, masking and OAF suppression. J. Acoust. Soc. Am. 81. 1043-1049. 